\title{
Visual and optometric issues with smart glasses in Industry 4.0 working environment
}

\author{
Vujica Herzog, N. ${ }^{a}{ }^{,}$, Buchmeister, B. ${ }^{a}$, Beharic, A. ${ }^{b}$, Gajsek, B. ${ }^{c}$ \\ ${ }^{a}$ University of Maribor, Faculty of Mechanical Engineering, Maribor, Slovenia \\ ${ }^{\mathrm{b}}$ Healthcare Center dr. Adolfa Drolca Maribor, Maribor, Slovenia \\ 'University of Maribor, Faculty of Logistics, Maribor, Slovenia
}

\section{A B S T R A C T}

Smart glasses are a kind of Head Mounted Display (HMD) with great potential in Industry 4.0 working environments, where shop floor workers must be supplied with critical information in a timely, accessible and safe manner to be as productive as possible. Smart glasses collect data from a wireless network and project it on a tiny screen before the user's eye. Despite several benefits, such as hands-free access to computer-generated info, routeing to storage locations, eliminating the need to carry handheld scanners or written documents, there are also possible problems evidenced from the literature. HMD can cause headaches, pressure in the eyes, problems with focusing and difficulties with text reading. To study the addressed problems, a research was performed together with Ophthalmologists from Maribor Healthcare Centre. The effects of using Vuzix M300 Smart glasses on users' comfort during order picking activities were researched in a testing warehouse environment at the Faculty of Mechanical Engineering, Maribor. The testing period lasts four hours. Several ophthalmologic tests (visual acuity, contrast sensitivity, visual field testing and colour test) were performed before and after use of smart glasses. Results show that there are some statistically significant differences before and after use of smart glasses in users' visual acuity and, surprisingly, a high percentage of scotomas in the right eye (where the projection of smart glasses was performed) after use of smart glasses that cannot be overlooked.
\end{abstract}

\section{ARTICLE INFO}

Keywords:

Head-mounted display (HMD);

Smart glasses;

Industry 4.0;

Warehouse;

Manual order picking system

*Corresponding author:

natasa.vujica@um.si

(Vujica Herzog, N.)

Article history:

Received 25 May 2018

Revised 19 November 2018

Accepted 23 November 2018

\section{References}

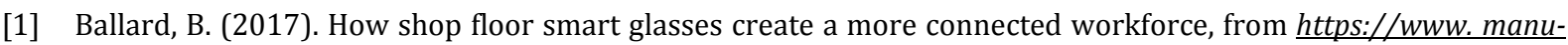
facturing.net/article/2017/01/how-shop-floor-smart-glasses-create-more-connected-workforce, accessed November 16, 2018.

[2] De Koster, R., Le-Duc, T., Roodbergen, K.J. (2007). Design and control of warehouse order picking: A literature review, European Journal of Operational Research, Vol. 182, No. 2, 481-501, doi: org/10.1016/i.ejor.2006. 07.009.

[3] Burinskiene, A. (2015). Optimising forklift activities in wide-aisle reference warehouse, International Journal of Simulation Modelling, Vol. 14, No. 4, 621-632, doi: 10.2507/IJSIMM14(4)5.312.

[4] Tompkins, J.A., White, J.A., Bozer, Y.A., Tanchoco, J.M.A. (2003). Facilities planning, Third edition, Hoboken, New Jersey, John Wiley, USA.

[5] Banduka, N., Veža, I., Bilić, B. (2016). An integrated lean approach to process failure mode and effect analysis (PFMEA): A case study from automotive industry, Advances in Production Engineering \& Management, Vol. 11, No. 4, 355-365, doi: 10.14743/apem2016.4.233.

[6] Gholamian, M.R., Heydari, M. (2017). An inventory model with METRIC approach in location-routing-inventory problem, Advances in Production Engineering \& Management, Vol. 12, No. 2, 115-126, doi: 10.14743/apem2017. $\underline{2.244}$. 
[7] Berlec, T., Kleindienst, M., Rabitsch, C., Ramsauer, C. (2017). Methodology to facilitate successful lean implementation, Strojniški Vestnik - Journal of Mechanical Engineering, Vol. 63, No. 7-8, 457-465, doi: 10.5545/sv-jme. 2017.4302.

[8] Dukic, G., Rose, L., Gajsek, B., Opetuk, T., Cajner, H. (2018). Space time and ergonomic assessment of orderpicking using vertical lift modules, In: Proceedings of 14th International Conference on Industrial Logistics, BenGurion University, Beer-Sheva, Israel, 68-74.

[9] Karabegović, I., Karabegović, E., Mahmić, M., Husak, E. (2015). The application of service robots for logistics in manufacturing processes, Advances in Production Engineering \& Management, Vol. 10, No. 4, 185-194, doi: 10.14743/apem2015.4.201.

[10] Dukić, G., Oluić, Č. (2004). Order-picking routing policies: Simple heuristics, advanced heuristics or optimal algorithm, Strojniški Vestnik - Journal of Mechanical Engineering, Vol. 50, No. 11, 530-535.

[11] Ames, B. (2017). Smart glasses get a second look from warehouses, from $h t t p: / / w w w . d c v e l o c i t y . c o m / a r t i c l e s /$ 20170213-smartglasses-get-a-second-look-from-warehouses/, accessed October 23, 2018.

[12] Peli, E. (1996). Visual and optometric issues with head-mounted displays, In: Proceedings of the IS\&T Optics \& Imaging in the Information Age, The Society for Imaging Science and Technology, Rochester, New York, 364-369.

[13] Eraslan, E., Can, G.F., Atalay, K.D. (2016). Mental workload assessment using a fuzzy multi-criteria method, Tehnički Vjesnik - Technical Gazette, Vol. 23, No. 3, 667-674, doi: 10.17559/TV-20140401112509.

[14] Klein-Theyer, A., Horwath-Winter, J., Rabensteiner, D.F., Schwantzer, G., Wultsch, G., Aminfar, H., Heidinger, A., Boldin, I. (2016). The impact of visual guided order picking on ocular comfort, ocular surface and tear function, PLoS One, Vol. 11, No. 6, doi: 10.1371/journal.pone.0157564.

[15] Josefsson, P., Lingegård, S. (2017). Potential of smart glasses in a spare parts distribution center, Master's thesis, Chalmers University of Technology, Gothenburg, Sweden.

[16] Schwerdtfeger, B., Reif, R., Gunthner, W.A., Klinker, G., Hamacher, D., Schega, L., Bockelmann, I., Doil, F., Tulmler, J. (2009). Pick-by-vision: A first stress test, In: Proceedings of IEEE International Symposium on Mixed and Augmented Reality, Orlando, Florida, USA, 115-124, doi: 10.1109/ISMAR.2009.5336484.

[17] Rammelmeier, T., Galka, S., Günthner, W.A. (2011). Active prevention of picking errors by employing pick-byvision, In: Proceedings of $4^{\text {th }}$ International Doctoral Students Workshop on Logistics, Otto-von-Guericke Universtät Magdeburg, Magdeburg, Germany, 79-83.

[18] Peli, E. (1990). Visual issues in the use of a head-mounted monocular display, Optical Engineering, Vol. 29, No. 8, 883-892, doi: 10.1117/12.55674.

[19] Peli, E. (1998). The visual effects of a head-mounted display (HMD) are not distinguishable from those of a desktop computer display, Vision Research, Vol. 38, No. 13, 2053-2066, doi: 10.1016/S0042-6989(97)00397-0.

[20] Schuff, D., Corral, K., Turetken, O. (2011). Comparing the understandability of alternative data warehouse schemas: An empirical study, Decision Support Systems, Vol. 52, No. 1, 9-20, doi: 10.1016/j.dss.2011.04.003.

[21] Han, J., Bae, S.H., Suk, H.-J. (2017). Comparison of visual discomfort and visual fatigue between head-mounted display and smartphone, In: Proceedings of the IS\&T International Symposium on Electronic Imaging, Human Vision and Electronic Imaging, Society for Imaging Science and Technology, 212-217, doi: 10.2352/ ISSN.24701173.2017.14.HVEI-146.

[22] Mocci, F., Serra, A., Corrias, G.A. (2001). Psychological factors and visual fatigue in working with video display terminals, Occupational \& Environment Medicine, Vol. 58, No. 4, 267-271, doi: 10.1136/oem.58.4.267.

[23] Cole, B.L., Maddocks, J.D., Sharpe, K. (1996). The Effect of VDUs on the eyes: A report of a 6-year epidemiological study, Optometry and Vision Science, Vol. 73, No. 8, 512-528, doi: 10.1097/00006324-199608000-00001.

[24] Blehm, C., Vishnu, S., Khattak, A., Mitra, S., Yee, R.W. (2005). Computer vision syndrome: A review, Survey of Ophthalmology, Vol. 50, No. 3, 253-262, doi: 10.1016/j.survophthal.2005.02.008.

[25] Rosenfield, M. (2011). Computer vision syndrome: A review of ocular causes and potential treatments, Ophthalmic \& Physiological Optics, Vol. 31, No. 5, 502-515, doi: 10.1111/j.1475-1313.2011.00834.x.

[26] Ramulu, P., Salim, S. (2017). Standard automated perimetry, from http://eyewiki.aao.org/Standard Automated Perimetry, accessed August 21, 2018.

[27] Parede, T.R.R., Torricelli, A.A.M., Mukai, A., Netto, M.V., Bechara, S.J. (2013). Quality of vision in refractive and cataract surgery, indirect measurers: Review article, Arquivos Brasileiros de Oftalmologia, Vol. 76, No. 6, 386-390, doi: 10.1590/S0004-27492013000600016.

[28] Karatepe, A.S., Köse, S., Eğrilmez, S. (2017). Factors affecting contrast sensitivity in healthy individuals: A pilot study, Turkish Journal of Ophthalmology, Vol. 47, No. 2, 80-84, doi: 10.4274/tjo.93763.

[29] Tepeš, M., Krajnik, P., Kopač, J. (2015). Framework proposition and technical guidelines for manufacturers of custom made tools, machinery and special equipment, Tehnički vjesnik - Technical Gazette, Vol. 22, No. 3, 581590, doi: 10.17559/TV-20140114133656.

[30] Veza, I., Mladineo, M., Gjeldum, N. (2016). Selection of the basic lean tools for development of croatian model of innovative smart enterprise, Tehnički Vjesnik - Technical Gazette, Vol. 23, No. 5, 1317-1324, doi: 10.17559/TV20160202120909.

[31] Işik, M.F., Haboğlu, M.R., Yilmaz, C., Yilmaz, E.N. (2018). Design and implementation of real-time monitoring and control system for distributed robotic systems supported with IOS/Android application, Tehnički Vjesnik - Technical Gazette, Vol. 25, No. 2, 423-428, doi: 10.17559/TV-20160125160226. 


\title{
Vpliv uporabe pametnih očal $v$ delovnem okolju Industrije 4.0 na vid uporabnika
}

\author{
Vujica Herzog, N. ${ }^{a,}{ }^{,}$, Buchmeister, B. ${ }^{a}$, Beharic, A. $^{b}$, Gajsek, B. ${ }^{c}$ \\ ${ }^{a}$ University of Maribor, Faculty of Mechanical Engineering, Maribor, Slovenia \\ ${ }^{\mathrm{b}}$ Healthcare Center dr. Adolfa Drolca Maribor, Maribor, Slovenia \\ 'University of Maribor, Faculty of Logistics, Maribor, Slovenia
}

\begin{abstract}
POVZETEK
Pametna očala so vrsta naglavnega prikazovalnika (Head Mounted Display HMD), ki ima velik uporabni potencial v različnih delovnih okoljih. Delujejo tako, da podatke, ki jih dobijo iz Wi-fi omrežja projicirajo na majhen zaslon pred očmi uporabnika. Kljub temu, da je znanih veliko prednosti uporabe pametnih očal, predvsem gre izpostaviti prostoročni način dela z brezžično povezavo do računalnika, odpade tudi prenašanje različnih skenerjev ali papirnatih dokumentov, je iz obstoječe literature možno zaslediti tudi nekaj težav povezanih z uporabo pametnih očal. Uporaba HMD naprav lahko povzroča glavobole, pritisk v očeh, probleme pri fokusiranju in težave pri branju besedila. Da bi preučili tovrstne težave, smo skupaj z okulisti iz Mariborskega zdravstvenega doma izvedli raziskavo. Učinke uporabe pametnih očal Vuzix M300 na udobje uporabnika med delom komisioniranja smo preučevali v testnem skladišču, ki smo ga postavili na Fakulteti za strojništvo Maribor. Perioda testiranja očal je trajala štiri ure. Pred in po uporabi pametnih očal smo izvedli več oftalmoloških testov, kot so pregled vidne ostrine, kontrastna senzitivnost, test vidnega polja in barvni test. Rezultati raziskave kažejo, da obstaja nekaj statistično pomembnih razlik med rezultati testov vidne ostrine, ki smo jih izvedli pred in po uporabi pametnih očal. Prav tako je bilo v desnem očesu (kjer je bila projekcija s pametnimi očali) po uporabi očal prisotnih večje število skotomov (temne lise na vidnem polju), kar je lahko resna težava za uporabnika in je ne bi smeli spregledati.
\end{abstract}

(C) 2018 CPE, University of Maribor. All rights reserved.
PODATKI O ČLANKU

Ključne besede:

Naglavni prikazovalnik;

Pametna očala;

Industrija 4.0;

Skladišče;

Ročni komisionirni sistem

*Kontaktna oseba: natasa.vujica@um.si

(Vujica Herzog, N.)

Zgodovina članka:

Prejet 25. maja 2018

Popravljen 19. novembra 2018

Sprejet 23. novembra 2018 